\title{
Erratum
}

\section{Personal orchestra: a real-time audio/video system for interactive conducting}

\author{
Jan Borchers $^{1}$, Eric Lee ${ }^{1}$, Wolfgang Samminger ${ }^{2}$, Max Mühlhäuser ${ }^{3}$ \\ ${ }^{1}$ Media Computing Group, Department of Computer Science, RWTH Aachen University, 52056 Aachen, Germany \\ e-mail: borchers@cs.rwth-aachen.de \\ 2 Telecooperation Department, University of Linz, 4040 Linz, Austria \\ e-mail: wolfgang.samminger@liwest.at; eric@cs.rwth-aachen.de \\ 3 Telecooperation Group, Darmstadt University, 64283 Darmstadt, Germany \\ e-mail: max@informatik.tu-darmstadt.de
}

Multimedia Systems 9: 458-465 (2004)

Due to an unfortunate error some corrections were not carried out:

- On page 460 paragraph 4.4, the first sentence should read: From continuously monitoring this incoming data, the current $x / y$ baton position as well as approximations for its first derivatives are known.

- On page 461 in the right-hand column line 9, the formula should read:

$2 \cdot \sqrt[12]{2}$, i.e. 2 semitones.

- On page 465 in the References, the http address in Ref. 1 should read: mpex.prosoniq.com/

- Also on page 465 in Ref. 3 by Borchers the title should read:

WorldBeat 